\title{
Deeply Virtual Compton Scattering and Nucleon Structure
}

\author{
M. Garçon \\ DAPNIA/SPhN, CEA-Saclay, 91191 Gif-sur-Yvette, France
}

\begin{abstract}
Deeply Virtual Compton Scattering (DVCS) is the tool of choice to study Generalized Parton Distributions (GPD) in the nucleon. After a general introduction to the subject, a review of experimental results from various facilities is given. Following the first encouraging results, new generation dedicated experiments now allow unprecedented precision and kinematical coverage. Several new results were presented during the conference, showing significant progress in this relatively new field. Prospects for future experiments are presented. The path for the experimental determination of GPDs appears now open.
\end{abstract}

Keywords: DVCS, Generalized Parton Distributions, Hard exclusive reactions

PACS: $12.38 .-t, 13.60 .-r, 14.20 . \mathrm{Dh}$

\section{INTRODUCTION}

The hard scattering of a virtual photon (produced by a high energy lepton beam) followed by the emission of a real photon from the target, is referred to as Deeply Virtual Compton Scattering, or DVCS. This process, $\gamma^{*} p \rightarrow \gamma p$, is characterized by four kinematical variables: the photon virtuality $Q^{2}$, the Bjorken variable $x_{B}$, the momentum transfer $t$ and the angle $\phi$ between the leptonic $\left(l l^{\prime} \gamma^{*}\right)$ and hadronic $\left(\gamma^{*} \gamma p\right)$ planes. It has been demonstrated that in the Bjorken limit (high $Q^{2}$ and fixed $x_{B}$ ) and for $|t| / Q^{2} \ll 1$, the leading (or twist-2) contribution to DVCS arises from the scattering from a single parton in the target nucleon and the subsequent rearrangement of this parton in the final state nucleon. This is referred to as the handbag diagram, which factorizes in a hard photon-parton scattering and a "soft" part characterizing the nucleon structure, the generalized parton distributions, or GPDs.

DVCS is one of the key reactions, and indeed the simplest one, to determine GPDs experimentally. Since GPDs offer a much richer conceptual framework for the understanding of nucleon structure, considerable interest has arisen in the determination of these distributions, and consequently in the measurement of the DVCS process.

Several recent reviews $[1,2,3,4]$ are indicative of the theoretical activity in this field. An introductory and heuristic presentation is given in Ref. [5].

\section{DVCS AND GPD}

The nucleon, as a spin 1/2 composite particle, is characterized by four chiral-even GPDs $H, \tilde{H}, E, \tilde{E}$ for each flavor $(u, d, s, g)$. Apart from their scale dependence $\left(Q^{2}\right)$, these distributions depend on 3 variables: $\xi=f\left(x_{B}\right)$, called skewedness, and $t$ are defined

\author{
CP870, Intersections of Particle and Nuclear Physics: $9^{\text {th }}$ Conference, \\ edited by T. M. Liss \\ (c) 2006 American Institute of Physics 978-0-7354-0368-0/06/\$23.00
}


TABLE 1. Summary of DVCS experiments. Only final results are quoted here in the references.

\begin{tabular}{|c|c|c|c|}
\hline Experiment & Observable & Non-dedicated runs & Dedicated runs \\
\hline H1 & $\sigma$ & {$[6,7]$} & - \\
\hline ZEUS & $\sigma$ & [8] & - \\
\hline \multirow[t]{3}{*}{ HERMES } & $\mathrm{BSA} / \mathrm{A}_{\mathrm{LU}}$ & [9] & 2006-2007 \\
\hline & BCA & {$[10]$} & 2006-2007 \\
\hline & TSA/A ${ }_{U L} \& A_{U T}$ & preliminary & - \\
\hline \multirow[t]{2}{*}{ CLAS } & BSA/A & [11] \& preliminary & $2005,2008 \& 11 \mathrm{GeV}$ \\
\hline & TSA/A & [12] & $2008 \& 11 \mathrm{GeV}$ \\
\hline \multirow[t]{2}{*}{ Hall A } & $\sigma \& \Delta \sigma_{\mathrm{LU}}$ & - & $2004[13] \& 11 \mathrm{GeV}$ \\
\hline & same on neutron & - & 2004 \\
\hline COMPASS & $\mathrm{BSA} / \mathrm{BCA}$ & - & in project (after 2010) \\
\hline
\end{tabular}

by the kinematics, while $x$, the average longitudinal momentum fraction of the parton between the initial and final states, appears only as an internal loop variable in the handbag diagram describing the DVCS process (the usual parton distributions $q(x)$ are recovered in the forward limit $\xi$ and $t \rightarrow 0$ ). The DVCS amplitude then contains terms of the form

$$
T^{D V C S} \sim \int_{-1}^{+1} \frac{H(x, \xi, t)}{x \pm \xi-i \varepsilon} d x+\cdots \sim \mathscr{P} \int_{-1}^{+1} \frac{H(x, \xi, t)}{x \pm \xi} d x-i \pi H( \pm \xi, \xi, t)
$$

Thus DVCS observables $(\sigma, \cdots)$ are in general expressed in terms of integrals over $x$ of the GPDs. However, many measured polarization observables single out the imaginary part of the amplitude, and are thus directly proportional to (a combination of) GPDs at the specific points $( \pm \xi, \xi, t)$. This situation arises because in the $l p \rightarrow l p \gamma$ reaction, the final state photon may be not only emitted by the target proton (DVCS), but also radiated by the incoming or outgoing leptons (Bethe-Heitler process, or BH). The DVCS-BH interference contains terms which can be uniquely extracted making use for example of beam or target polarization.

\section{DVCS EXPERIMENTS}

All data concerning DVCS on the nucleon is summarized in Table 1. Most of the existing data set originates from non-dedicated experiments and does not have the statistical accuracy allowing to extract GPDs from measurements. However the available data is already in semi-quantitative agreement with existing GPD models, even at moderate values of $Q^{2}$. This opens the way to a rich phenomenology concerning the extraction of GPDs.

All experiments had new data to report at this conference, and this talk included the presentation of results from the first dedicated DVCS experiments, performed at Jefferson Lab, in Hall A with unprecedented precision and in CLAS with unprecedented kinematical coverage. 

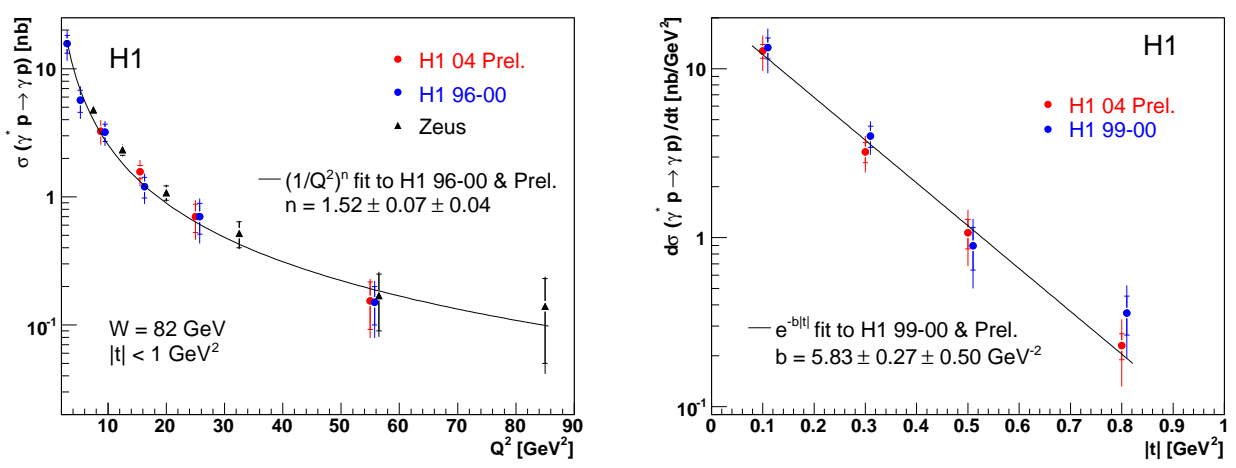

FIGURE 1. Compiled HERA results [14]: $Q^{2}$ and $t$ dependence of reduced $\gamma^{*} p \rightarrow \gamma p$ (DVCS) cross sections.

\section{DESY/H1 \& ZEUS}

At very high energies, and thus very low $x_{B}$, DVCS may probe the gluon GPDs in the nucleon. Both H1 and ZEUS at HERA extracted DVCS cross sections from $e p \rightarrow e \gamma X$ data $[6,8]$, and $\mathrm{H} 1$ was able to measure $t$-distributions as well after including the detection of a remnant proton: $e p \rightarrow e \gamma p X$ [7]. The total statistics was recently doubled [14]. The data is integrated over $\phi$, thus cancelling BH-DVCS interference terms; the calculated BH cross sections, as well as simulated inclusive background, are subtracted. Overall systematic errors are estimated of the order of $25 \%$.

The $Q^{2}$ and $t$ dependences of the DVCS cross sections are shown in Fig. 1. The fitted values of $Q^{2}$ and $W$ slopes are in reasonable agreement with values expected from the phenomenology of hard scattering processes. GPD calculations (NLO-QCD) reproduce fairly well the data. Within this formalism, there is no need for intrinsic skewedness ( $\xi$ dependence) which is generated solely by the GPD evolution. Alternatively, the data may also be reproduced by a color dipole model. The significance of this dual interpretation is yet to be explored.

\section{DESY/HERMES}

At the HERMES fixed (internal) target experiment, at a beam energy of $27.6 \mathrm{GeV}$, the DVCS process is up to now identified through the analysis of $e p \rightarrow e \gamma X$ events. The missing mass resolution does not allow for the sole selection of exclusive events. Inelastic contributions have to be evaluated by Monte Carlo methods, and observables are studied as a function of an inelasticity cut. The results suffer somewhat from the relatively low luminosity. Nervertheless, taking full advantage of the polarized and unpolarized targets, of the availability of both electron and positron beams, several interesting DVCS observables have been and are being explored. The various asymmetries all result 
from the interference between $\mathrm{BH}$ and DVCS processes:

- The beam spin asymmetry [9] exhibits the characteristic $\sin \phi$ behaviour expected from BH-DVCS interference.

- Final results for the beam charge asymmetry were made available just at the time of this conference [10]. This asymmetry is mostly sensitive to an integral over $x$ of the GPD $H$. The results are indeed suggestive of the anticipated $\cos \phi$ behaviour.

- Transversely polarized target spin asymmetries are particularly sensitive to the GPD $E$, and thus to the quark angular momentum [15]. They were used to extract, in a model-dependent way, an estimate of $J_{u}+J_{d}$ [16], albeit with large statistical and systematic uncertainties.

\section{JLab/CLAS}

In several data sets from CLAS, using a polarized beam and an unpolarized target, $e p \rightarrow e p X$ events were analyzed in order to extract the contribution of exclusive $e p \rightarrow$ ep $\gamma$ events to the beam spin asymmetry (BSA or $A_{\mathrm{LU}}$ ). At $4.2 \mathrm{GeV}$ [11] and 4.8 $\mathrm{GeV}$ [17], $e p \gamma$ and $e p \pi^{0}$ contributions may be separated through an analysis of the missing mass $\left(M_{X}\right)$ spectrum. At $5.75 \mathrm{GeV}$ [18], the $\pi^{0}$ contribution is estimated through a (model-dependent) Monte Carlo simulation. At small values of $|t|$, the $\pi^{0}$ contribution remains small (less than $10 \%$ ) and beam spin asymmetries are being extracted in a large kinematical range in $Q^{2}$ and $x_{B}$.

Using a longitudinally polarized target, DVCS target spin asymmetries (TSA or A $\mathrm{UL}_{\mathrm{L}}$ ) could be extracted as well, nicely illustrating the enhanced sensitivity of this observable (with respect to BSA) on the GPD $\tilde{H}$ [12].

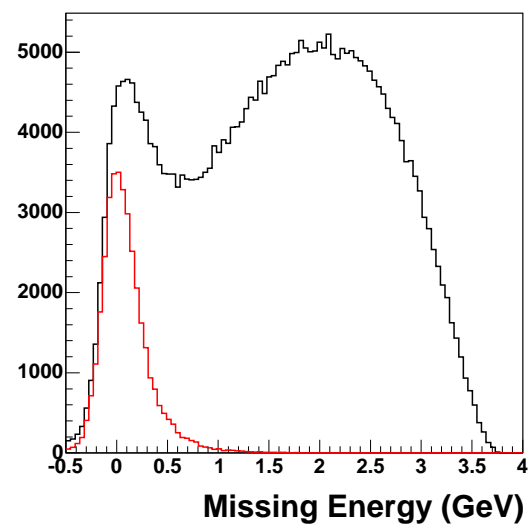

FIGURE 2. Histogram of missing energy $E_{X}$ in the reaction $e p \rightarrow e p \gamma X$, from the CLAS/DVCS experiment with the new inner calorimeter. In black, for all measured ep $\gamma X$ configurations. In grey (red online), after kinematical cuts on missing transverse momentum and on photon angles. The exclusive $e p \rightarrow e p \gamma$ reaction is thus well identified [19]. 
A dedicated DVCS experiment was installed and ran successfully in the first semester of 2005. In order to ensure a better selection of exclusive $e p \rightarrow e p \gamma$ events, all three final-state particles were detected. A new inner calorimeter was added to the standard configuration of CLAS in order to cover the photons emitted between 5 and 15 degrees in the laboratory. This calorimeter was built from 424 lead-tungstate crystals read by avalanche photodiodes and stabilized in temperature. Based on a technology developed for LHC, it is the first of its kind to operate successfully in a physics experiment. The intense background originating mostly from Møller electrons was focused along the beam axis by a specially designed two-coil supraconducting solenoid surrounding the liquid hydrogen target. The $e p \rightarrow e p \gamma$ events show up as a clear peak once kinematical constraints are applied to all events where one electron, one proton and one photon are detected (Fig. 2). Even then, a residual contamination of $\pi^{0}$ events, of the order of a few percents, is still possible.

Preliminary results [19] were shown at the conference, but are not presently available for circulation. The statistics and kinematical coverage are such that the data can be binned in $15\left(x_{B}, Q^{2}\right)$ bins, 5 bins in $t$ and 12 in $\phi$. This data set will likely have a big impact on all studies of kinematical dependences of the beam spin asymmetries $A_{L U}$, and will constrain significantly any GPD model or parametrization.

\section{JLab/Hall A}

In the fall of 2004, a dedicated DVCS experiment was performed in Hall A. While the scattered electron was detected in the standard High Resolution Spectrometer, the outgoing photon and proton were detected in specially designed detectors (a leadfluoride calorimeter with fast digitizing electronics for the former, a plastic scintillator annular array for the latter). This highly performant set-up allowed for operation at a luminosity of $10^{37} \mathrm{~cm}^{-2} \mathrm{~s}^{-1}$, even with the calorimeter in direct view of the liquid hydrogen target. Three kinematical settings were used, at fixed $x_{B}$ and increasing values of $Q^{2}$. Thanks to the excellent HRS and calorimeter resolutions, the $e p \rightarrow e p \gamma$ events could be identified clearly in the $e p \rightarrow e \gamma X$ missing mass spectra, the proton array being used only to calibrate the shape of these distributions. Moreover, the precision given by a small aperture magnetic spectrometer and a kinematically matched calorimeter allowed to measure cross sections, that is $\Delta \sigma_{\mathrm{LU}}\left(=d^{4} \Sigma\right.$ in Fig. 3), the numerator of the above mentioned BSA, and $\sigma$. The results give the first direct evidence of scaling in DVCS. That is, Compton scattering is really occuring at the quark level and higher-twist effects are small. Purely experimental extraction of GPDs can really begin [13].

A slightly modified set-up was used in a subsequent run on a deuterium target, in order to extract quasi-free DVCS on the neutron. BSA and $\Delta \sigma_{\mathrm{LU}}$ are expected to be especially sensitive to the GPD E. Preliminary results [20] do exhibit an asymmetry of opposite sign as for the proton, as expected from this sensitivity to $E$. 


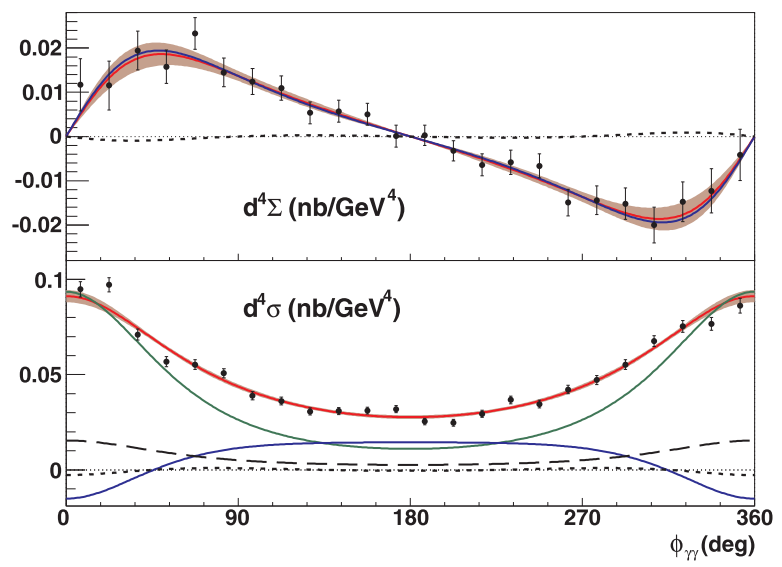

FIGURE 3. Hall A results for helicity-dependent and helicity-independent cross sections, at $x_{B}=0.35$, $Q^{2}=2.3 \mathrm{GeV}^{2}, t=-0.28 \mathrm{GeV}^{2}$, as a function of $\phi$. The curves are contributions to fits described in Ref. [13].

\section{OTHER HARD EXCLUSIVE REACTIONS}

Though the focus of this talk has been DVCS, it should be reminded here that hard exclusive leptoproduction of mesons is a necessary complement to DVCS in order to determine independent GPD combinations and obtain a flavor decomposition of these distributions [5]. Scaling (in other words the dominance of the handbag diagram) is expected to occur at higher $Q^{2}$ than in the case of DVCS. Experimental studies of the reactions $e p \rightarrow e p \pi / \omega / \rho / \Phi$ have been performed or are underway at HERMES, CLAS and COMPASS. In the case of the $\rho$ meson, qualitative agreement with GPD models may be found, but the theory does not allow at this stage a phenomenological extraction of GPDs from this data. To reach such a quantitative stage in exclusive meson production may require the higher values of $Q^{2}$ that will be accessible with the $12 \mathrm{GeV}$ energyupgrade of the CEBAF accelerator at Jefferson Lab.

Another reaction of primary interest in this field is "double" DVCS $\left(\gamma^{*}(q) p \rightarrow\right.$ $\left.\gamma^{*}\left(q^{\prime}\right) p\right)$, where the final state photon is virtual $\left(q^{\prime} \neq 0\right)$ and materializes through a lepton pair. Preliminary studies with CLAS data showed the difficulty in extracting DDVCS from identified $e p \rightarrow e p e^{+} e^{-}$events [21]. This remains an exciting challenge for future experiments.

\section{FUTURE EXPERIMENTS}

The continuation and the next generation of DVCS experiments are being actively pursued:

- HERMES is presently commissioning a (proton) recoil detector and will dedicate its running time in 2006-2007 to measurements of BSA and BCA in $e p \rightarrow e p \gamma$. 
- As the CEBAF accelerator will resume its $6 \mathrm{GeV}$ operation in 2008, the dedicated CLAS/BSA experiment will double its present statistics and a new CLAS/TSA experiment will take place. Both use the small inner calorimeter described above, and the longitudinally polarized $\mathrm{NH}_{3}$ target will be used for the first high-statistics measurement of the target spin asymmetry.

- COMPASS at CERN is presently making plans and tests to build a recoil detector that would enable the measurement of DVCS with a 100-200 GeV polarized muon beam $\left(\mu^{+}\right.$and $\left.\mu^{-}\right)$[22]. Asymmetries combining the beam charge and the beam polarization have been shown to be very sensitive to the GPD input in a kinematical domain where gluons and sea quarks play a large role. It is hoped that such an experiment would run as of 2010.

- One of the main focus of the CEBAF $12 \mathrm{GeV}$ upgrade at JLab is precisely the study of GPDs. DVCS will remain the tool of choice, but it is anticipated that the high $Q^{2}$ reached in the valence region will allow for complementary (and essential) studies of deeply virtual and exclusive meson production. The first round of proposals in this direction have just been approved by the CEBAF PAC. They include DVCS in CLAS12 - the upgraded CLAS - and in Hall A, as well as hard pseudoscalar meson production in CLAS12. The energy-upgraded beam is expected to be available for physics in 2014 [23].

\section{CONCLUSION AND PERSPECTIVES}

The experimental and theoretical activity related to DVCS and GPDs is very intense, some ten to twelve years after the realization that GPDs lead to a rich, powerful and unifying concept of nucleon structure. In this talk, I have classified the experiments in two categories: non-dedicated (or first generation) and dedicated experiments. The latter are likely to represent a significant qualitative and quantitative step toward the extraction of GPDs from experiments. Early attempts to do so are already in progress, but the methods need to be refined. Theoreticians and experimenters are working together toward this goal.

In parallel, progress was made in lattice QCD, which allows the calculation of GPD moments and, from a parameterization and extrapolation of these moments, the calculation of GPDs themselves (see e.g. Ref. [24]).

Experiments, lattice calculations, and constrained GPD parametrizations lead in particular to the emerging picture where valence quarks form the nucleon core while seaquarks and gluons (see e.g. the recent analysis of H1 data [25]) are more peripheral. Unravelling precisely how these spatial distributions evolve with parton longitudinal momenta (see e.g. Ref. [26]) is one of the main goals of these studies.

Scaling in DVCS is now shown to occur at very modest values of $Q^{2}$. The next generations of experiments at Jefferson Lab (before and after the upgrade), DESY and CERN will make full use of this for a quantitative determination of GPDs. 


\section{Answer to a question during plenary talk}

I did not answer quite satisfactorily a question at the end of my talk, related to the kinematical minimum value of $|t|$. At the large values of $x_{B}$ which are accessible at $\mathrm{JLab}$, and will be even more so after the $12 \mathrm{GeV}$ upgrade, $|t|_{\min }$ is becoming sizeable: $|t|_{\min } \simeq 0.4 \mathrm{GeV}^{2}$ at $x_{B} \simeq 0.5,0.7 \mathrm{GeV}^{2}$ at $x_{B} \simeq 0.6$, nearly independent of $Q^{2}$ for $Q^{2}$ sufficiently large. The question then arises about the determination of GPDs at large values $x_{B}$ as $t \rightarrow 0$ relevant for the $\mathrm{Ji}$ integral yielding the angular momentum. First, large values of $x$ (the argument of GPDs) will be probed even at small $x_{B}$ in observables sensitive to the real part of the DVCS amplitude $(\sigma, \mathrm{BCA}$, measurements of BSA precise enough to constrain the denominator of the asymmetry, etc...). Second, it is anticipated - but not proven yet - that a global fit of observables with the most general parameterization of GPDs will allow for a significant extrapolation into the unphysical region $t \rightarrow 0$ for all values of $x$ and $\xi$. Whenever the recoil proton detection is required on fixed-target experiments, there is an experimental threshold for $|t|$ : presently at 0.09 $\mathrm{GeV}^{2}$ with CLAS, it should reach around $0.01 \mathrm{GeV}^{2}$ with the HERMES recoil detector and $0.04 \mathrm{GeV}^{2}$ with CLAS12.

\section{ACKNOWLEDGMENTS}

I acknowledge the help of Harut Avakian, Nicole d'Hose, Frank Ellinghaus, FrançoisXavier Girod, Franck Sabatié and Laurent Schoeffel in gathering information and graphs concerning the quoted experiments.

\section{REFERENCES}

1. M. Diehl, Phys. Rep. 388, 41 (2003).

2. X. Ji, Ann. Rev. Nucl. Part. Sci 54, 413 (2004).

3. P. Kroll, Prog. Part. Nucl. Phys. 55, 281 (2005).

4. A.V. Belitsky and A.V. Radyushkin, Phys. Rep. 418, 1 (2005).

5. M. Garçon, Eur. Phys. J. A 18, 389 (2003).

6. C. Adloff et al. (H1 collaboration), Phys. Lett. B517 (2001) 47.

7. A. Aktas et al. (H1 collaboration), Eur. Phys. J. C 44 (2005) 1.

8. S. Chekanov et al. (ZEUS collaboration), Phys. Lett. B573 (2003) 46.

9. A. Airapetian et al. (HERMES collaboration), Phys. Rev. Lett. 87 (2001) 182001.

10. A. Airapetian et al. (HERMES collaboration), hep-ex/0605108, submitted to Phys. Rev. Lett.

11. S. Stepanyan et al. (CLAS collaboration), Phys. Rev. Lett. 87 (2001) 182002.

12. S. Chen et al. (CLAS collaboration), Phys. Rev. Lett. 77 (2006) 072002.

13. C. Muñoz Camacho et al. (Jefferson Lab Hall A collaboration), nucl-ex/0607029, submitted to Phys. Rev. Lett.

14. B. Roland (for the H1 collaboration), hep-ex/0605096, to appear in Proc. of 14th Int. Workshop on Deep Inelastic Scattering (DIS 2006), Tsukuba, Japan, April 2006; P. Marage, these proceedings.

15. X.-D. Ji, Phys. Rev. Lett. 78 (1997) 610.

16. F. Ellinghaus (for the HERMES collaboration), these proceedings.

17. G. Gavalian et al., in preparation.

18. H. Avakian et al., analysis in progress.

19. F.-X. Girod et al., analysis in progress.

20. M. Mazouz et al., analysis in progress. 
21. M. Garçon, $2^{\text {nd }}$ Int. Workshop on the Structure of the Nucleon at Large Bjorken $x$ (HiX2004), A.I.P. Conf. Proc. 747 (2005) 173.

22. Expression of Interest : CERN-SPSC-2005-007, SPSC-EOI-005, January 17, 2005.

23. L. Elouadhriri, these proceedings.

24. M. Gockeler et al. (QCDSF Collaboration), Phys. Rev. Lett. 92 (2004) 042002; Nucl. Phys. A755 (2005) 537.

25. D. Müller, hep-ph/0605013.

26. M. Burkardt, Phys. Lett. B595 (2004) 245. 